\title{
Remaining Lifetime Modelling for Replacement of Power Transformer Populations
}

\author{
A. van Schijndel ${ }^{1 *}$, J.M. Wetzer ${ }^{2}$ and P.A.A.F. Wouters ${ }^{1}$ \\ ${ }^{1}$ Eindhoven University of Technology, The Netherlands \\ ${ }^{2}$ KEMA, Arnhem, The Netherlands \\ *E-mail : a.v.schijndel@tue.nl
}

\begin{abstract}
The age of the majority of power transformers applied in the western electricity network varies between 25 and 50 years. Depending on the load history and time of operation, replacement on short term is imminent. A technically sound policy concerning the replacement of these assets must be based on knowledge of (i) the life expectancy or reliability of individual components, (ii) how these failure probabilities cumulate to a replacement wave, and (iii) how to manage an expected replacement wave.

The population reliability is obtained from individual transformer reliabilities using Arrhenius based modelling of paper insulation degradation. This modelling technique includes measures to cope with inherent uncertainties in available data. Population reliability figures are obtained using an adapted kout-of-N failure model. The modelling method is applied to existing populations of power transformers in The Netherlands, to evaluate their expected replacement wave.
\end{abstract}

Index Terms--Life estimation, Maintenance, Paper insulation, Power system reliability, Power transformers, Power transformer insulation, Reliability modeling.

\section{NOMENCLATURE}

$A$ pre-exponential reaction constant

$D P$ degree of polymerisation

$E_{a}$ activation energy ( $\left.\mathrm{J} / \mathrm{mole}\right)$

$F_{i} \quad$ individual failure probability

$k \quad$ reaction rate $\left(\mathrm{s}^{-1}\right)$

$p_{d p} \quad$ DP distribution function

$P_{t h} \quad$ DP threshold

$R \quad$ universal gas constant $(\mathrm{J} / \mathrm{mol} \cdot \mathrm{K})$

$R_{i} \quad$ individual reliability

$R^{(k, N)}$ probability of at least $k$ out of $N$

$R_{P} \quad$ population reliability

$T$ absolute temperature $(\mathrm{K})$

$\theta_{a}$ ambient temperature $\left({ }^{\circ} \mathrm{C}\right)$

$\theta_{h} \quad$ hotspot temperature $\left({ }^{\circ} \mathrm{C}\right)$

\section{INTRODUCTION}

$\mathrm{T}$ HE ageing of power systems and their components are of great concern in terms of reliability and investments. To guarantee required quality at acceptable cost, it is essential to base decisions on a reliable forecast of future behaviour. The

This work is supported by KEMA Nederland B.V. and the Dutch utilities Essent Netwerk B.V. and Eneco Netbeheer B.V. present work is dedicated to a population of power transformers. This is especially relevant since many power transformers that are installed in the past are expected to reach their end-of-life in the coming decades resulting in a replacement wave.

The work presented forms part of a research project aiming at integral transformer reliability models including different fail mechanisms. This paper is restricted to paper insulation degradation based on the DP (degree of polymerisation) value. But the generic approach chosen in this project allows other mechanisms to be included later.

In previous work we have presented a modelling approach for individual transformers [1], [2], based on the mechanical strength of the paper insulation. Starting from these individual reliability distributions (section III), a method for forecasting the population reliability from the individual reliabilities is described (section IV). The technique is applied to populations of transformers of two utilities in The Netherlands (section $\mathrm{V}$ ). The ultimate goal is to provide an optimisation tool for maintenance replacement scenarios.

\section{INDIVIDUAL RELIABILITY}

The individual reliability is determined in a three step process and is based on the mechanical strength of the transformer paper insulation. In the first step the degree of polymerisation and its threshold value at which failure can occur are determined. Next, the uncertainties of these values are incorporated. In the third step the reliability and its uncertainty is calculated.

\section{A. Determining the DP-value}

The temperature dependency of the DP-value is given by the Arrhenius relation, this is extensively discussed by Emsley, Lundgaard and others in [3]-[5]. This leads to the following relation:

$$
\frac{1}{D P(t)}-\frac{1}{D P(0)}=\int_{0}^{t} k(\tau) d \tau,
$$

where $D P(t)$ is the DP-value at time $t, D P(0)$ is the initial DP value and $k(t)$ is the time dependent reaction rate. The time dependent reaction rate has the Arrhenius form

$$
k=A \exp \left(-\frac{E_{a}}{R T}\right)
$$


where $E_{a}$ is the molar activation energy, $R$ the universal gas constant, $A$ is a process constant and $T$ is the temperature in Kelvin. The activation energy $E_{a}$ and process constant $A$ can still vary with time, because the physical properties of the paper can change. For the temperature in (2), the hotspottemperature of the IEC loading guide [6] is used. The hotspottemperature is given by

$$
\theta_{h}=\theta_{a}+\Delta \theta_{o r}\left(\frac{1+r K^{2}}{1+r}\right)^{x}+H g_{r} K^{y}
$$

where $K$ is the time dependent relative load per unit. The other input parameters are: oil exponent $x$; winding exponent $y$; loss ratio $r$; ambient temperature $\theta_{a}$; Hot-spot to top-oil gradient $H g_{r}$; and top-of-tank oil rise $\Delta \theta_{o r}$.

\section{B. Uncertainty Estimation}

For determining the uncertainty the error estimation technique is used, this will result for (2) in

$$
\frac{\Delta k}{k}=\sqrt{\left(\frac{\Delta A}{A}\right)^{2}+\left(\frac{\Delta E_{a}}{R T}\right)^{2}+\left(\frac{E_{a} \Delta T}{R T^{2}}\right)^{2}},
$$

where the $\Delta$ 's indicate the uncertainties in the corresponding variables.

\section{Probability Calculation}

The reliability is an integration of all possible DP-values, indicated with $v$, according to

$$
R_{i}(t)=1-\int P_{t h}(v) p_{d p}(v, t) d v
$$

Here $p_{d p}$ is the distribution function of the DP-value and $P_{t h}$ the probability that the transformer has failed with a specific DP-value. In this case we use truncated normal distributions with mean $\mu$ equal to the DP-value on time $t$ and $2 \sigma$ equal to uncertainty value defined in the previous subsection. For the DP-threshold these values are 250 and 50 for $\mu$ and $2 \sigma$, respectively [1], [2].

\section{Population Reliability Model}

The individual reliability results, as described in the previous section, can be used to obtain the reliability of a transformer population. The method assumes that the individual reliabilities, $R_{i}$, and consequently the failure probabilities $F_{i}=1-R_{i}$, are uncorrelated. The probability that exactly $j$ transformers from a population of $N$ fail, and the probability that less than $k$ failures have occurred at time $t$ are given by [2]

$$
\begin{gathered}
P_{F}^{(j)}(t)=\sum_{i_{1}=1}^{N} F_{i_{1}} \sum_{i_{2}>i_{1}}^{N} F_{i_{2}} \Lambda \sum_{i_{j}>i_{j-1}}^{N} F_{i_{j}} \prod_{i \neq\left\{i_{1} \Lambda i_{j}\right\}}^{N} R_{i}(t), \\
R^{(N-k+1, N)}(t)=\sum_{j=0}^{k-1} P_{F}^{(j)}(t) .
\end{gathered}
$$

The superscript $(N-k+1, N)$ in (6b) indicates that at least $N-k+1$ out of $N$ transformers are still operational. It is clear that with large populations $N$ and significant numbers of failures $k$ a direct evaluation of (6) is elaborate, due to the enormous number of possible combinations. Only if (subsets of) transformers have identical individual reliabilities it can be applied with success, because a binomial factor can then replace (part of) the summations and multiplication.

A far more efficient approach is to apply a pivotal decomposition by considering the following recurrent relation:

$$
R^{(i, j)}=\left(1-R_{j}\right) \cdot R^{(i, j-1)}+R_{j} \cdot R^{(i-1, j-1)}
$$

The probability that at least $i$ out of a set of $j$ transformers are operational is equal to the probability that the "last" transformer $j$ has failed and from the remaining $j-1$ at least $i$ transformers work, plus the probability that transformer $j$ is in working order and a maximum of $i-1$ of the rest are operational. The begin and end condition of (7) are $R(0, j)=1$ and $R(j+1, j)=0$. This algorithm reduces the analysis for a system of up-to $k$ failures from a population of $N$ transformers to the order of $N \times k$ operations [7]. Moreover, the results for up to $k-1, k-2$, etc. are directly available as intermediate results of the recurrent analysis.

From (7) the mean-time-to-failure (MTTF) of the failure of $k$ transformers, $M T T F_{k}$, can be written as

$$
M T T F_{k}=\int_{0}^{\infty} R^{(N-k+1, N)}(t) d t .
$$

Equation (8) links the time with the population reliability. The population reliability $R_{P}$ belonging to the $M T T F_{k}$ is defined as

$$
R_{P}\left(t=M T T F_{k}\right)=\frac{N-k}{N} .
$$

\section{Reliabilty of Populations FROM Two Utilities}

The bar graph of Fig. 1 displays the installation times of the power transformers of two Dutch utilities. The total number of installed power transformers is 579. The big investment wave between 1960 and 1980 is clearly visible. In this section the two populations, shown in Fig. 1 will be used as input for the population reliability model presented in the previous section.

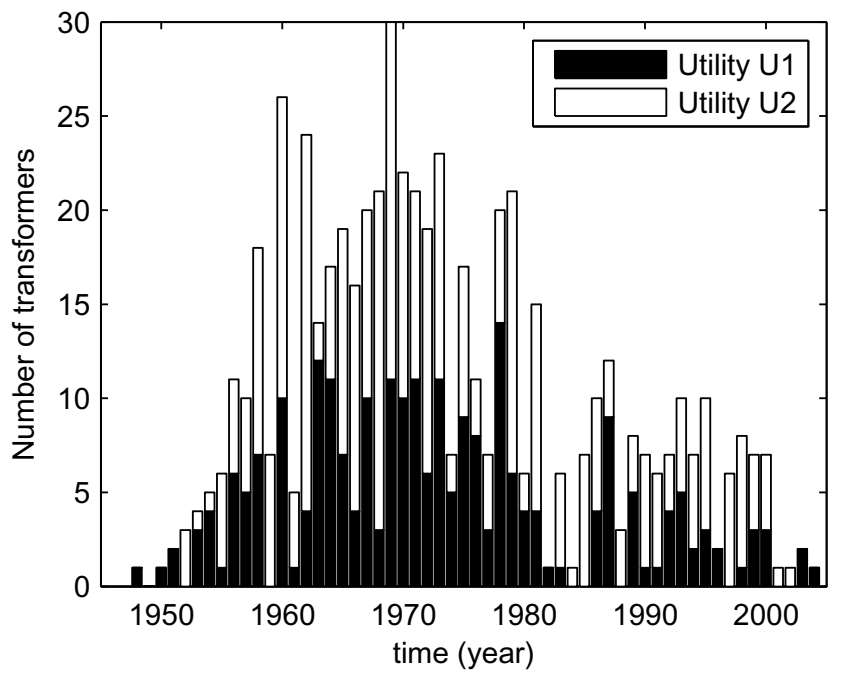

Fig. 1. Transformers installed per year, which are still in operation in two Dutch utilities. Utility U1 and U2 own 242 and 337 power transformers, respectively. 
From these populations, next to the installation time, the cooling modes: Oil-Natural-Air-Natural (ONAN), OilNatural- Air-Forced (ONAF), Oil-Forced (OF) and OilDirected (OD) are used as input parameters. For the ambient temperature, $\theta_{a}$, the temperature model of the IEC loadingguide [8] is applied, given as

$$
\begin{aligned}
\theta_{a}(t)=\theta_{a y} & +A_{1} \cos \left[\frac{2 \pi}{24 \cdot 365}\left(t-24 \varphi_{1}\right)\right] \\
& +A_{2} \cos \left[\frac{2 \pi}{24}\left(t-\varphi_{2}\right)\right]
\end{aligned}
$$

with time $t$ in hours. The constants in (10) are $\theta_{a y}=9.4{ }^{\circ} \mathrm{C}$, $A_{1}=7.4{ }^{\circ} \mathrm{C}, \varphi_{1}=199, \mathrm{~B}=13{ }^{\circ} \mathrm{C}$ and $\varphi_{2}=15$ hours. The average yearly load growth is currently $2 \%$, but in our calculation a load increase of $1.5 \%, 2 \%$ and $2.5 \%$ will be investigated. To obtain the per-unit load, the average for every hour in the period 2003-2006 is calculated. This average is normalised to a per-unit load of 0.4 .

\section{A. Utility U1}

Utility U1 has a population of 242 power transformers with average installation date of 1973 and a standard deviation of 12 years. This combined with the information of (10) results in the projected replacement wave shown in Fig. 2. For 1.5\% annual load growth the mean value of the retirement date is 2079 with a standard deviation of 8 years. The values belonging to a $2 \%$ annual load increase has an average retirement year of 2062, with a standard deviation of 5 years. The $2.5 \%$ annual increase resulted in a mean of 2053, and standard deviation of 5 years.

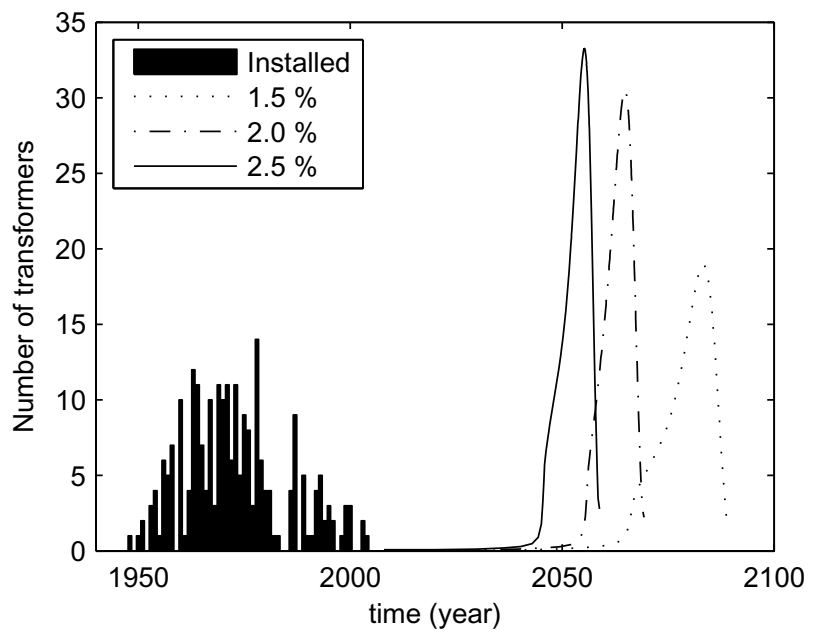

Fig. 2. Installation times of utility $\mathrm{U} 1$, and the expected replacement waves for a load growth of $1.5 \%, 2 \%$ and $2.5 \%$; the total number of installed transformers for utility U1 is 242 .

\section{B. Utility U2}

The 337 power transformers of utility U2 have an average install date of 1974, with a standard deviation of 13 years. The replacement wave curves for this utility are plotted in Fig. 3. The retirement date for $1.5 \%$ is 2079 , with a standard deviation of 8 years. For $2 \%$ and $2.5 \%$ we find 2062 and 2052, respectively, both with standard deviations of 5 years.

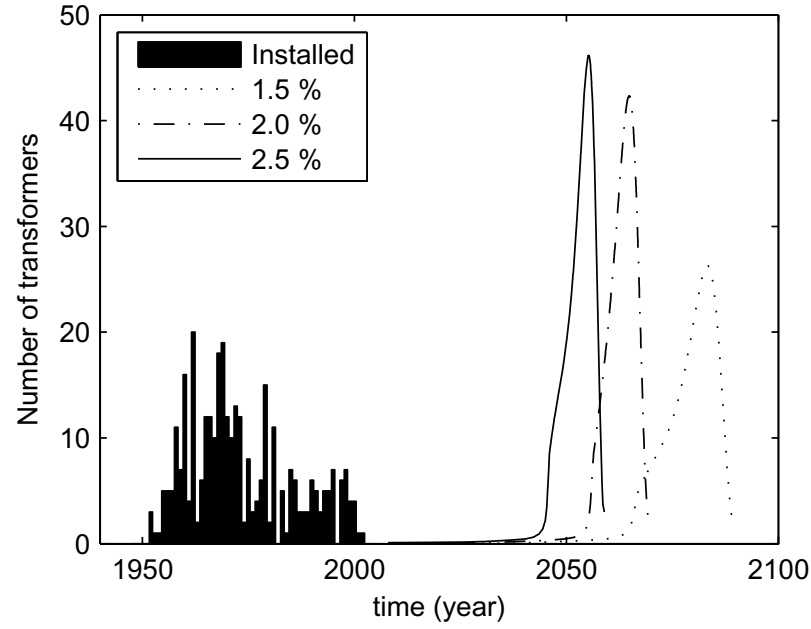

Fig. 3. Installation times of utility $\mathrm{U} 2$, and the expected replacement waves for a load growth of $1.5 \%, 2 \%$ and $2.5 \%$; the total number of installed transformers for utility $\mathrm{U} 2$ is 337 .

\section{DISCUSSION AND CONCLUSION}

The individual reliabilities based on the paper degradation model of Section III can be transformed with the model of Section IV into population reliability without too much computational effort. The results for the two utilities, shown in Fig. 2 and Fig. 3, are quite similar, because of comparable past policy of both utilities. The sensitivity of the prediction to the forecasted increase of the average load arises, because of the non-linear age acceleration with temperature, and therefore with load. The ageing is exponentially correlated with load and the load is assumed to grow exponentially. Hence, small deviations in future scenarios with respect to the expected load will have a large effect on the projected fail behaviour. This can be illustrated by varying the starting base-load as is shown in Fig. 4. The position of the replacement wave shifts clearly as a function of this parameter. The probabilistic model presented in this paper enables to analyse the effect of planned scenarios for transformer populations.

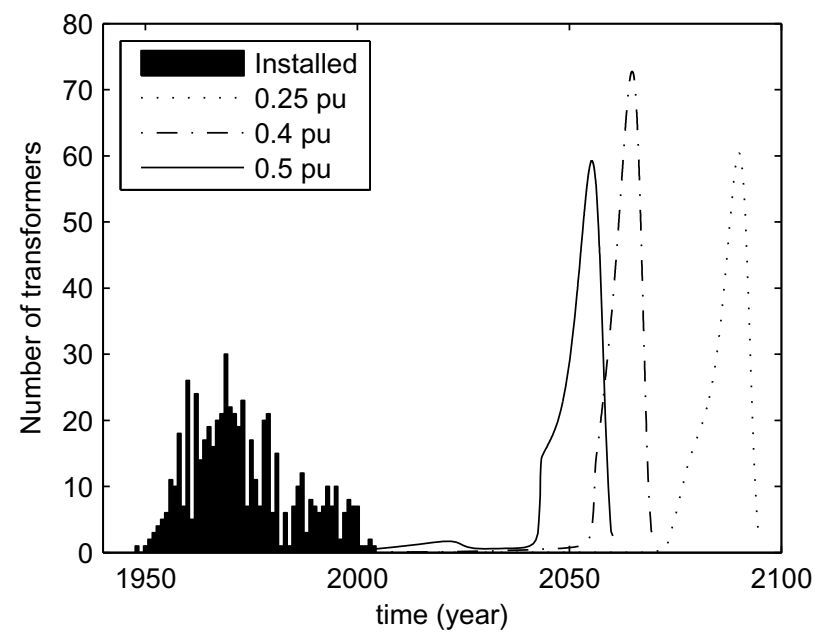

Fig. 4. Installation times of both utility, with the expected replacement waves for a maximum load of $0.25 \mathrm{pu}, 0.4 \mathrm{pu}$ and $0.5 \mathrm{pu}$. The total number of installed transformers for both utility is 579 and the load increase is kept constant at $2 \%$. 
In predicting the replacement wave more realistically, the scenarios put into the model should incorporate:

- Real load values based on the location of the transformer. The model input parameters were chosen to illustrate the model capabilities.

- The effect of maintenance or failure of a transformer on others; if in a substation maintenance is performed on one transformer, another transformer will take the load of both transformers. Due to strong non-linearity of ageing with load the time spans with double load, although they may be short, can have a pronounced effect on the life expectancy.

In this paper the individual transformer reliability is limited to degradation of the mechanical strength of the paper insulation. This is only one of the possible failure mechanisms. To get better insight in the coming replacement wave, the individual reliability model should include other failure modes, such as degradation of the oil, the bushings and the tapchanger.

\section{ACKNOWLEDGMENT}

The research presented is made possible by the financial support and active participation of KEMA, Essent Netwerk and Eneco Netbeheer. We gratefully acknowledge the contributions of Harry Verhaart, Maarten Berende and Herman Arts.

\section{REFERENCES}

[1] A. van Schijndel, J. M. Wetzer, and P. A. A. F. Wouters, "Forecasting transformer reliability," in 2006 IEEE Conference on Electrical Insulation and Dielectric Phenomena IEEE Cat No. 06CH37829. IEEE, Oct 2006, pp. 577-582.

[2] A. van Schijndel, J. M. Wetzer, and P. A. A. F. Wouters, "Forecasting reliability of transformer populations," in 19th International Conference on Electricity Distribution. CIRED, 2007.

[3] A. M. Emsley and G. C. Stevens, "Review of chemical indicators of degradation of cellulosic electrical paper insulation in oil-filled transformers," IEE Proceedings Science, Measurement and Technology, vol. 141, no. 5, pp. 324-334, 1994.

[4] L. E. Lundgaard, W. Hansen, D. Linhjell, and T. J. Painter, "Aging of oil-impregnated paper in power transformers," IEEE Transactions on Power Delivery, vol. 19, no. 1, pp. 230-239, 2004.

[5] "Cigré 323, ageing of cellulose in mineral-oil insulated transformers," Report, 2007.

[6] "IEC 60076-7 Power transformers - Part 7: Loading guide for oilimmersed power transformers," Report, 2005.

[7] W. Kuo and M. J. Zou, Optimal reliability modeling. Hoboken, New Jersey, USA: John Wiley \& Sons, Inc., 2003.

[8] "IEC 60354 loading guide for oil-immersed power transformers," Report, 1991. 